\title{
Single nucleotide polymorphisms of cytokine-related genes and association with clinical outcome in a Chagas disease case-control study from Brazil
}

\author{
Lucia Elena Alvarado-Arnez', Angelica Martins Batista', Silvia Marinho Alves², Gloria Melo², \\ Virgínia Maria Barros de Lorena ${ }^{3}$, Cynthia C Cardoso ${ }^{4}$, Isabela Resende Pereira', Cristina Carrazzone ${ }^{2}$, \\ Antonio G Pacheco ${ }^{5}$, Wilson Oliveira Jr², Milton Ozório Moraes ${ }^{6}$, Joseli Lannes-Vieira ${ }^{1 /+}$ \\ ${ }^{1}$ Fundação Oswaldo Cruz-Fiocruz, Instituto Oswaldo Cruz, Laboratório de Biologia das Interações, Rio de Janeiro, RJ, Brasil \\ ${ }^{2}$ Ambulatório de Doença de Chagas e Insuficiência Cardíaca do Pronto Socorro Cardiológico de Pernambuco, Recife, PE, Brasil \\ ${ }^{3}$ Fundação Oswaldo Cruz-Fiocruz, Instituto Aggeu Magalhães, Departamento de Imunologia, Laboratório de Imunoparasitologia, Recife, PE, Brasil \\ ${ }^{4}$ Universidade Federal do Rio de Janeiro, Instituto de Biologia, Departamento de Genética, Laboratório de Virologia Molecular, Rio de Janeiro, \\ RJ, Brasil \\ ${ }^{5}$ Fundação Oswaldo Cruz-Fiocruz, Programa de Computação Científica, Rio de Janeiro, RJ, Brasil \\ ${ }^{6}$ Fundação Oswaldo Cruz-Fiocruz, Instituto Oswaldo Cruz, Laboratório de Hanseníase, Rio de Janeiro, RJ, Brasil
}

BACKGROUND The severity of chronic chagasic cardiomyopathy (CCC), the most frequent clinical outcome of Chagas disease (CD), has been associated with cytokine-enriched heart tissue inflammation, and high serum levels of transforming growth factor (TGF $\beta$ ), interferon-gamma (IFN $\gamma$ ), and tumour necrosis factor (TNF). Conversely, increased interleukin (IL)-10 serum concentrations have been associated with asymptomatic CD. Cytokines and cytokine-related gene polymorphisms may control cytokine expression and have been proposed to contribute to CCC outcomes.

OBJECTIVES We evaluated the association of 13 cytokine-related genes (TGFB: rs8179181, rs8105161, rs1800469; IL10: rs1800890, rs1800871, rs1800896; IFNG: rs2430561; TNF: rs1800629; BAT1: rs3853601; LTA: rs909253, rs2239704; TNFR1: rs767455; TNFR2: rs1061624) with risk and progression of CCC.

FINDINGS Four hundred and six seropositive patients from CD endemic areas in the state of Pernambuco, north-eastern Brazil, were classified as non-cardiopathic (A, 110) or cardiopathic (mild, B1, 163; severe, C, 133). We found no evidence of TGFB, IL10, TNF, or TNFR1/2 gene polymorphisms associated with CCC risk or progression. Only BAT1 rs3853601 $-22 \mathrm{G}$ carriers (B1 vs. $\mathrm{C}: \mathrm{OR}=0.5 ; \mathrm{p}$-value $=0.03)$ and $I F N G$ rs2430561 +874AT $(\mathrm{A} v s . \mathrm{C}: \mathrm{OR}=0.7 ; \mathrm{p}$-value $=0.03 ; \mathrm{A} v s . \mathrm{B} 1+\mathrm{C}: \mathrm{OR}=0.8 ; \mathrm{p}$-value $=0.02$ ) showed a significant association with protection from cardiopathy in a logistic regression analysis with adjustment for gender and ethnicity; however, the association disappeared after performing adjustment for multiple testing. A systematic review of $T N F$ rs $1800629-308 \mathrm{G}>\mathrm{A}$ publications included five studies for meta-analysis (534 CCC and 472 asymptomatic patients) and showed no consensus in pooled odds ratio (OR) estimates for A allele or A carriers $(\mathrm{OR}=1.4$ and $1.5 ; \mathrm{p}$-values $=0.14$ and 0.15 , respectively). In CD patients, TNF serum levels were increased, but not affected by the TNF rs1800629-308A allele.

MAIN CONCLUSIONS Our data suggest no significant contribution of the analysed gene variants of cytokine-related molecules to development/severity of Chagas' heart disease, reinforcing the idea that parasite/host interplay is critical to CD outcomes.

Key words: Chagas disease - Trypanosoma cruzi - cardiomyopathy - cytokines - gene polymorphism - meta-analysis

Chagas disease (CD), a neglected disease caused by the protozoan parasite Trypanosoma cruzi, afflicts $\sim 10$ million in Latin America (Herricks et al. 2017). In the last decades, due to immigration, T. cruzi-infected individuals have raised the attention of people working in

doi: 10.1590/0074-02760170489

Financial support: FAPERJ (CNE/E-26/101.549/2010;

E-26/110.153/2013; E-26/111.709/2013; CNE/E-26/202.969/2015); CNPq (474234/2012-6-Universal; INCTV, National Institute for Science and Technology for Vaccines; DECIT/MS/CNPq 403979/2012-9; BPP 4296073664961416; PROEP/IOC/CNPq 400146/2011).

JL-V and MOM are research fellows of CNPq and CNE/FAPERJ; LEA-A was supported by the Graduate Students Program multinational scholarship

PEC-PG and as a postdoctoral fellow by the funding CAPES.

LEA-A, AMB and SMA contributed equally to this work.

+ Corresponding author: lannes@ioc.fiocruz.br

LEAA's current address: Franz Tamayo University/UNIFRANZ

(Cochabamba, Bolivia).

Received 10 November 2017

Accepted 5 March 2018 the healthcare systems of North America and Europe (Herricks et al. 2017). Acute infection is mostly asymptomatic, and 10-30 years after infection $60-70 \%$ of infected individuals show no clinical signs, remaining in the indeterminate form of CD. Nevertheless, $20-30 \%$ of these $\mathrm{CD}$ patients developed the cardiac form of $\mathrm{CD}$, chronic chagasic cardiomyopathy (CCC), which shows a spectrum of severity from mild to severe, with heart tissue inflammation and remodelling, fibrosis, electrical and structural abnormalities, and thromboembolic events, culminating in heart failure (Rassi Jr et al. 2017). The pathogenic determinants of this range of clinical manifestations remain unclear. Genetic factors may determine the form of the disease and severity of the cardiac form (Williams-Blangero et al. 2011). Several studies have implicated parasite load and/or diversity, as well as the systemic inflammatory profile with enrichment in cytokines and inflammatory mediators, as determinants of the severity of CD (Cunha-Neto and Chevillard 2014, Rassi Jr et al. 2017). As a theoretical framework, 
we assumed that clinical manifestation and severity of Chagas' heart disease are multifactorial processes related to (i) parasite persistence and (ii) an inappropriate or dysregulated host immune response, which may result from genetic predisposition and/or interplay between the immune system and the parasite. In the present study, we will focus on the role of cytokines and cytokine-related gene polymorphisms as contributing factors for the development and severity of Chagas' heart disease.

In CD patients, serum concentrations of transforming growth factor (TGF)- $\beta$ have been associated with heart fibrosis intensity, disease progression, and mortality (Saraiva et al. 2013). Several studies have revealed that high tumour necrosis factor (TNF) levels in serum are associated with the development and severity of the cardiac form of CD (Sousa et al. 2014). High IL-10 expression has been observed in patients with the indeterminate form of $\mathrm{CD}$, but not in patients with the cardiac form (Sousa et al. 2014), supporting a lack of immunoregulatory machinery in CCC patients.

Cytokines play crucial roles in the regulation of immune responses. Gene polymorphisms may determine differential cytokine expression, which can contribute to clinical features, ranging from the development of immunological disorders to susceptibility to infections or disease severity. Several variants in different genes have been studied in T. cruzi-infected South American populations, mostly Brazilians, Colombians, and Peruvians. The most commonly studied single nucleotide polymorphisms (SNPs) are TNF, LTA, BATI, TGFB, and IL10, and, although some associations with risk or progression of Chagas' heart disease have been described, there is a need to replicate the findings in different populations (Beraún et al. 1998, Rodríguez-Pérez et al. 2005, Ramasawmy et al. 2006, Drigo et al. 2007, Calzada et al. 2009, Costa et al. 2009, Flórez et al. 2011, Pissetti et al. 2013).

In $\mathrm{CD}$, association studies of cytokine gene polymorphisms are restricted in number, frequently have a small number of patients, and, mostly, are not conclusive regarding the development/progression of the cardiac form of disease as an outcome. The present study was designed to determine whether SNPs in key molecules in the immune response to $T$. cruzi infection and immunoregulation (Cunha-Neto and Chevillard 2014), namely TGFB (rs8179181, rs8105161, rs1800469), IL10 (rs1800890, rs1800871, rs1800896), interferon-gamma (IFNG) (rs2430561), TNF (rs1800629), and its receptors TNFR1/2 (TNFR1: rs767455; TNFR2: rs1061624), LTA (rs909253, rs2239704), and BAT1 (rs3853601), are associated with the development and progression of CCC. We focused our study on a group of T. cruzi-seropositive patients from the northeast region of Brazil that have not been evaluated in previous studies for $\mathrm{CD}$ and genetic polymorphisms. Patients from the state of Pernambuco were classified in cardiomyopathy stages accordingly to the I Latin American Guideline for the Diagnosis and Treatment of Chagas' Heart Disease (Andrade et al. 2011). Further, we summarise previously reported studies for the $T N F$ rs 1800629 $-308 \mathrm{G}>\mathrm{A}$ polymorphism to obtain a consensus association estimation by means of meta-analysis.

\section{MATERIALS AND METHODS}

Study subjects - A group of 406 patients that attended to the Ambulatório de Doença de Chagas e Insuficiência Cardíaca do Pronto Socorro Cardiológico de Pernambuco (PROCAPE)/Universidade do Estado de Pernambuco (UPE) were enrolled (July 2010 - June 2015) in an unmatched case-control study. The recruited patients had serological diagnoses of $\mathrm{CD}$ based on at least two positive tests: enzyme-linked immunosorbent assay (ELISA), western blotting, and/or indirect immunofluorescence, performed by the Central Reference Laboratory (LACEN) of Pernambuco, Brazil, in accordance with the 1st (2005) and 2nd (2015) Brazilian Consensus on Chagas Disease (Dias et al. 2016). At baseline, the patients were evaluated by anamnesis, and 12-lead electrocardiography findings were recorded (ECG; Ecafix, São Paulo, SP, Brazil). Two-dimensional and M-mode echocardiography (ECHO) doppler was performed using a Vivid 3 (GE Health Care, Wauwatosa, WI, USA), and digital images were recorded. Participants were classified into three (A, B1, and C) different clinical groups accordingly to the I Latin American Guideline for the Diagnosis and Treatment of Chagas' Heart Disease (Andrade et al. 2011). Patients under 18 years of age or with digestive or cardiodigestive forms of $\mathrm{CD}$ and co-infections were excluded. The study, with the number of patients included in each clinical group, had $76 \%$ statistical power to detect a genetic association of 1.85 , estimated with the lower minor allele frequency of 0.10 under the additive model.

DNA extraction and SNP genotyping - Genomic DNA was isolated from frozen blood samples using a precipitation salting out technique. After extraction, DNA was quantified using a NanoDrop ND-1000 Spectrophotometer (NanoDrop Technologies, USA). Altogether, we analysed 13 SNPs using TaqMan assays [Supplementary data (Table I)]. Reactions were performed with $30 \mathrm{ng}$ of yield DNA for each sample following the manufacturer's recommendations for allelic discrimination using a ViiA 7 Real Time polymerase chain reaction (PCR) System (Applied Biosystems, USA). SNPs were analysed for the cluster IL10-TGFB1 and IFNG: rs1800890, rs1800871, rs1800896, rs8179181, rs8105161, rs1800469, and rs2430561; the cluster TNF-BAT-LTA: rs1800629, rs3853601, rs909253, and rs2239704; and the cluster TNFR1-TNFR2: rs767455 and rs1061624. Raw genotyping data were deposited at https:// www.arca.fiocruz.br/handle/icict/20995.

Functional analysis - Serum levels of TNF were quantified using ELISA DuoSet (R\&D Biosystems, Minneapolis, MN) according to manufacturer's instructions. Data were analysed (GraphPad Prism software version 5.0 for Windows) by comparing the levels of cytokines: (i) correlating with the left ventricular ejection fraction (LVEF; \%); (ii) between the different clinical groups; and (iii) stratifying cytokine levels according to TNF-308 SNP genotypes. For this study, 10 triatomineexposed and region-matched individuals excluded from our genetic study because of negative $T$. cruzi serology were included as non-infected controls. 
TABLE I

Demographic, epidemiological and clinical data of the recruited patients

\begin{tabular}{|c|c|c|c|c|}
\hline Variable $^{a}$ & $\begin{array}{l}\text { A Group } \\
\mathrm{N}=110\end{array}$ & $\begin{array}{c}\text { B1 Group } \\
\mathrm{N}=163\end{array}$ & $\begin{array}{l}\text { C Group } \\
\mathrm{N}=133\end{array}$ & p-value \\
\hline \multicolumn{5}{|l|}{ Gender } \\
\hline Female & $71(64.5)$ & $126(77.3)$ & $85(63.9)$ & \\
\hline Male & $39(35.5)$ & $37(22.7)$ & $48(36.1)$ & \\
\hline Age $(\text { years })^{\mathrm{b}}$ & $51 \pm 12$ & $60 \pm 13$ & $60 \pm 11$ & $\mathrm{KW}<0.001$ \\
\hline$\leq 45$ & $37(33.6)$ & $23(14.4)$ & $17(12.8)$ & \\
\hline$>45$ & $73(66.4)$ & $138(85.7)$ & $116(87.2)$ & \\
\hline Ethnicity $(\mathrm{n}=353)$ & 94 & 141 & 118 & Chisq. 0.34 \\
\hline White & $27(28.7)$ & $26(18.4)$ & $27(22.9)$ & \\
\hline Black & $8(8.5)$ & $11(7.8)$ & $13(11.0)$ & \\
\hline Mestizo & $59(62.8)$ & $104(73.8)$ & $78(66.1)$ & \\
\hline Monthly income $(\mathrm{n}=377)$ & 106 & 149 & 122 & Chisq. 0.82 \\
\hline Up to $1 \mathrm{MW}$ & $70(66.7)$ & $99(66.5)$ & $88(72.1)$ & \\
\hline $2-4$ & $14(13.3)$ & $23(15.4)$ & $14(11.5)$ & \\
\hline More than 5 & $21(20.0)$ & $27(18.1)$ & $20(16.4)$ & \\
\hline Education $(n=379)$ & 103 & 154 & 122 & Chisq. 0.14 \\
\hline Up to 4 years & $84(81.6)$ & $123(79.9)$ & $108(88.5)$ & \\
\hline More than 4 years & $19(18.4)$ & $31(20.1)$ & $14(11.5)$ & \\
\hline Region $(\mathrm{n}=358)$ & 101 & 153 & 121 & Chisq $<0.001$ \\
\hline PE, Agreste & $25(24.8)$ & $22(14.4)$ & $31(25.6)$ & \\
\hline PE, Mata & $21(20.8)$ & $79(51.6)$ & $52(43.0)$ & \\
\hline PE, Metropolitan & $6(5.9)$ & $8(5.2)$ & $6(5.0)$ & \\
\hline PE, Sertão & $27(26.7)$ & $31(20.3)$ & $14(11.6)$ & \\
\hline Other States & $22(21.8)$ & $13(8.5)$ & $18(14.9)$ & \\
\hline Clinical information & 110 & 163 & 133 & \\
\hline $\operatorname{LVEF}(\%)^{\mathrm{b}}$ & $67 \pm 5$ & $66 \pm 6$ & $40 \pm 11$ & $\mathrm{KW}<0.001$ \\
\hline Benznidazole & $49(44.5)$ & $19(11.6)$ & $19(14.3)$ & \\
\hline
\end{tabular}

$a$ : results are presented as number (frequency); $b$ : mean \pm standard deviation; $\mathrm{n}$ : number of patients; MW: minimum wage; PE: state of Pernambuco; LVFE: left ventricular ejection fraction.

Statistical analysis - Analyses were performed in R environment version 3.3.3 using the following packages: coin, epiDisplay, gap, genetics, stats, haplo.stats, meta, metafor, and SNPassoc. The comparison amongst demographic or clinical variables was performed either by Chi-square or Kruskal-Wallis test when appropriate, and the frequencies of each variable excluded missing data. Genotypic and minor allele carrier frequencies were determined, and associations with $\mathrm{CD}$ were evaluated by means of unconditional logistic regression comparing individuals at different stages of the cardiac form of CD disease. Two non-genetic variables, gender and ethnicity, were included as categorical covariates in the regression model. First, we tried to determine the influence of SNPs on developing the cardiac form of CD. For that, patients from group A were considered controls, and patients from $\mathrm{B} 1$ and $\mathrm{C}$ groups were considered cases. Then, to examine the influence of SNPs on severity of CD cardiomyopathy, people in group B1 (mild $\mathrm{CCC}$ ) were considered controls, and people in group $\mathrm{C}$ (severe CCC) were considered cases. Allele-dose effects were tested with the Cochran-Armitage trend test. Also, the false discovery rate (FDR) was determined to adjust for multiple testing in the different genetic models. For polymorphisms located within the same gene, haplotype frequencies were estimated by the maximum likelihood method and compared using the same unconditional regression models as used for the analysis for individual SNPs. A linkage disequilibrium (LD) analysis, evaluated trough $\mathrm{r}^{2}$ statistics, was performed using Haploview software separately for TGFB1, IL10, and TNF-BAT-LTA clusters. Finally, the analysis of TNF serum levels was performed using GraphPad Instat software version 3.0 (San Diego, CA). Statistical significance was considered with $\mathrm{p}$-values $<0.05$, with a Bonferroni correction applied when ANOVA tests were performed.

Meta-analysis - Published articles that evaluated the association between $\mathrm{CD}$ and gene polymorphisms were identified in databases such as MEDLINE using PubMed (http://www.ncbi.nlm.nih.gov/pubmed) and the Cochrane library (http://www.cochranelibrary.com/). The search keywords were a combination of: "cytokines" and "Chagas disease", "polymorphism(s)" and "SNP(s)". In addition, when evaluating each article in- 
dividually, we reviewed reference lists and related citations suggested by PubMed to broaden our results. We did not use SNP rs identification numbers in the search. As inclusion criteria, we considered studies if they were published up to March 2017 and provided sufficient genotypic data to determine allelic counts for the analysis. Studies were excluded if they were related to a previous publication or if the control individuals deviated from Hardy-Weinberg equilibrium (HWE).

Two authors (LEAA and AMB) independently extracted genotype counts from the studies that met the inclusion criteria [Supplementary data (Fig. 1)]. The recorded information included variables such as first author, year in which the study was published, population that was evaluated, age and number of females and males for both cases and controls, source of control individuals, genotyping method, and, finally, genotype counts for cases and controls.

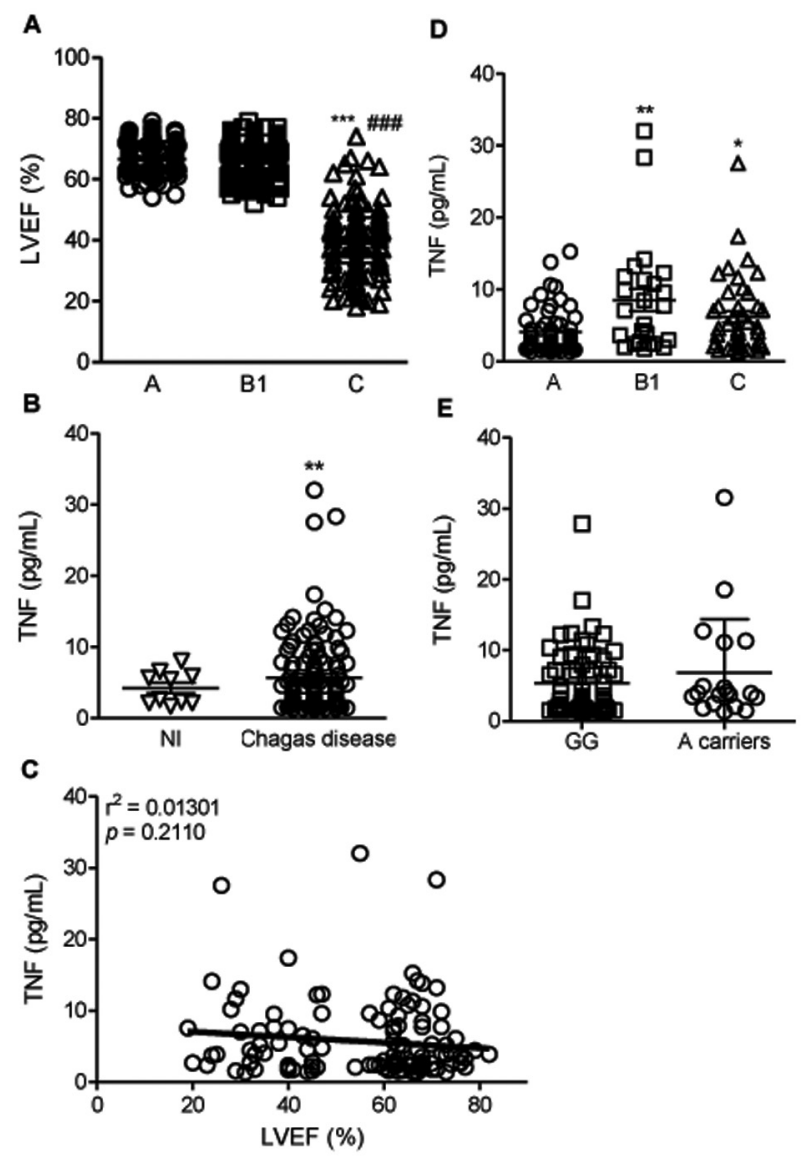

Fig. 1: TNF levels and association with left ventricular ejection fraction (LVEF) and genotypes in Trypanosoma cruzi-infected patients. (A) LVEF of CD patients grouped as A (asymptomatic), B1 (mild CCC),

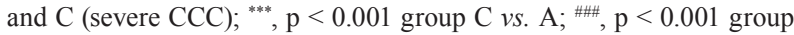
C vs. B1 (Kruskal-Wallis test). (B) TNF levels in the sera of subjects who were seronegative and considered non-infected (NI) and those that were seropositive for Chagas disease; ${ }^{* *}, \mathrm{p}<0.01$ ( $t$-student test). (C) Correlation between TNF serum concentrations and LVEF in patients; $p=0.2110, r^{2}=0.01301$ (linear regression). (D) TNF concentrations in the sera of CD patients grouped as A (asymptomatic), B1 (mild CCC), and $\mathrm{C}$ (severe $\mathrm{CCC}$ ); ${ }^{*}, \mathrm{p}<0.05,{ }^{* *}, \mathrm{p}<0.01$ group $\mathrm{C}$ and $\mathrm{B} 1 v s$. A (ANOVA, Bonferroni post-test). (E) Comparison of subjects according to the presence or absence of allele A (Mann-Whitney test).
We used a Chi-square test to determine whether genotype frequencies in the control groups of each of the selected studies followed HWE. Publication bias was evaluated by Egger's test to provide statistical evidence for funnel plot symmetry. Heterogeneity across studies was established by Cochran's Q statistic. Pooled OR estimates were obtained by DerSimonian and Laird random effects model referencing to the minor allele for each polymorphism. Forest plots represented individual OR values for each study and pooled ORs also referring to the minor allele for the studied SNPs. To determine the effect of each study on the overall OR, we performed a sensitivity analyses in which individual studies were removed sequentially. Adjustment for environmental effects and population stratification were not performed due to the lack of such covariates. The statistical calculations were carried out in $R$.

Ethics - Signed informed consents were obtained from all patients included in the study. All study protocols were approved by the Ethics Committees of Fiocruz/RJ (541/09) and PROCAPE/UPE (80210/10).

\section{RESULTS}

Epidemiological data and clinical classification of the enrolled patients - The main characteristics of the enrolled individuals are shown in Table I. The recruited individuals were classified as stage A, a group of $110 \mathrm{pa}-$ tients without cardiac symptoms and with normal ECG and ECHO; stage B1, a group of 163 patients with structural cardiopathy, evidenced by ECG or ECHO changes, but with normal global ventricular function and neither current nor previous signs and symptoms of congestive heart failure (CHF); and stage C, a group of 133 patients with ventricular dysfunction and current or previous symptoms of CHF. Mean age and standard deviation for each of the clinical groups were as follows: A (51 \pm $12)$, B1 $(60 \pm 13)$, and $C(60 \pm 11)$, although more than $65 \%$ of patients in either group were in the $>45$-yearold stratum. The distribution of patients according to gender showed a high frequency of females in groups A (64.5\%), B1 (77.3\%), and C (63.9\%). Regarding ethnicity, most of the patients identified themselves as mestizo. Independent of the clinical stage, most of the patients had a monthly income of up to one minimum wage (MW) and an education level of up to four years. As an important clinical variable, the LVEF was similar in patients of group A $(67 \% \pm 5 \%)$ and group B1 $(66 \% \pm 6 \%)$, but showed a significant decrease in patients of group $\mathrm{C}$ $(40 \% \pm 11 \%$, p-value $<0.001)$, as shown in Table I and Fig. 1A. The use of the trypanocidal drug benznidazole was registered in $44.5 \%$ of the patients in stage A, $11.6 \%$ in stage B1, and $14.3 \%$ in stage C (Table I).

Polymorphisms at TGFB, IFNG, IL-10, TNF, LTA, and BATl genes and association with Chagas' heart disease - A call rate efficiency of $90 \%$ was obtained for all polymorphisms studied. The study of the rs 8179181 $(+20743 \mathrm{C}>\mathrm{T}), \mathrm{rs} 8105161(+19318 \mathrm{~A}>\mathrm{G})$ and rs1800469 (-509 C $>\mathrm{T})$ polymorphisms at $T G F B$ gene showed no significant association when comparing susceptibility to or severity of CCC (Table II). Similarly, the IL10 SNPs 


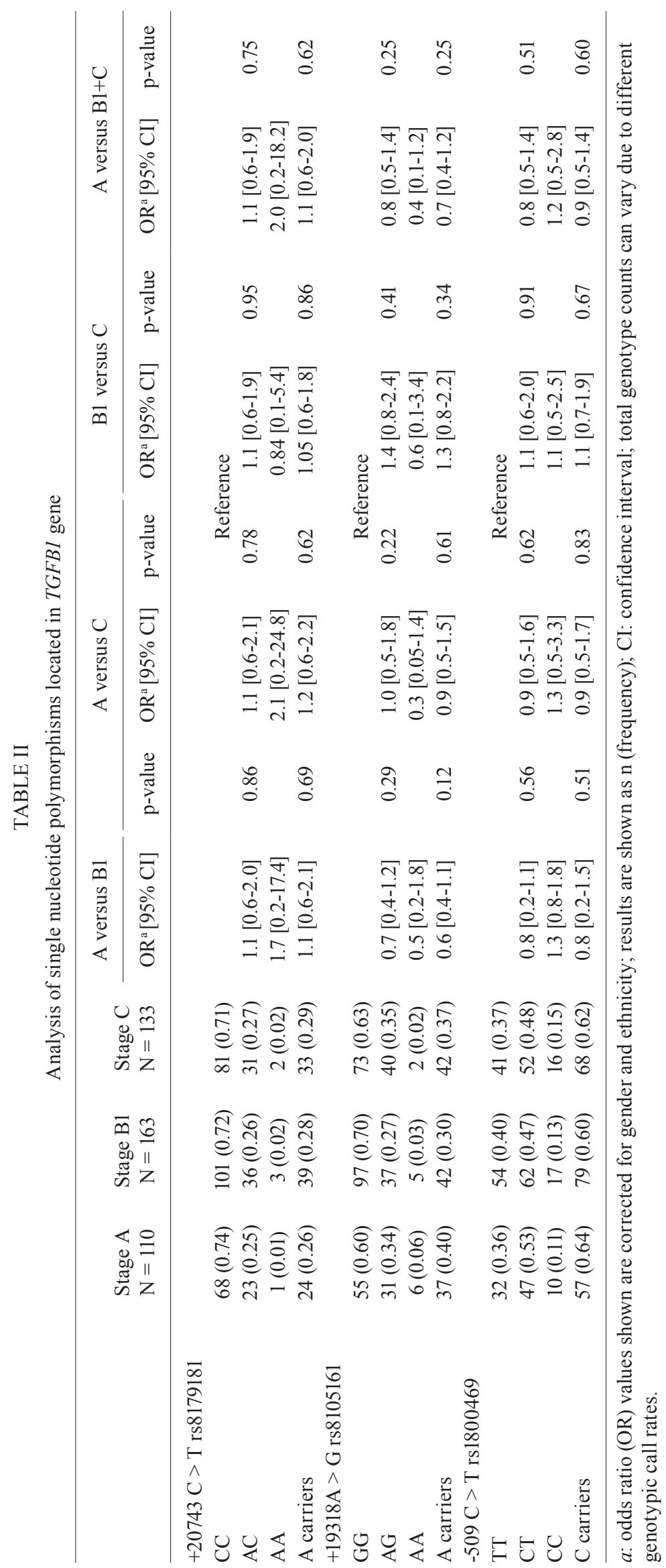


rs1800890 (-3575 T>A), rs1800896 (-1082 G>A), and rs1800871 (-819 C $>\mathrm{T})$ did not act as a genetic modifier of Chagas' heart disease outcome (Table III). Interestingly, the IFNG SNP rs2430561 (+874 T>A) showed a significant association with protection to CCC development, when comparing non-cardiopathic (A) vs. mildcardiopathic $(\mathrm{B} 1)$ patients that were genotype AT $(\mathrm{OR}=$ $0.7, P$-value $=0.03)$ and $\mathrm{A}$ carriers $(\mathrm{OR}=0.6, \mathrm{p}$-value $=$ $0.06)$ and non-cardiopathic (A) vs. cardiopathic $(\mathrm{B} 1+\mathrm{C})$ patients that were genotype $\mathrm{AT}(\mathrm{OR}=0.8, \mathrm{p}$-value $=$ $0.02)$ and A carriers $(\mathrm{OR}=0.6$, $\mathrm{p}$-value $=0.06)$, as described in Table III. Total genotype counts could vary because of differences in genotypic call rates.

As shown in Table IV, out of the four studied SNPs harboured in the TNF-BATI-LTA cluster, only BATI rs3853601 -22 CG genotype and $\mathrm{G}$ allele carriers showed an association with protection $\left(\mathrm{OR}_{\mathrm{CG}}=0.8, \mathrm{p}\right.$-value $=$ 0.05 and $\mathrm{OR}_{\mathrm{G}}=0.5$, p-value $\left.=0.03\right)$ when analysing cardiomyopathy severity, comparing B1 patients as a control group and $\mathrm{C}$ patients as a case group. However, the significance of both associations (IFNG rs2430561 and $B A T 1$ rs3853601) was lost after performing adjustment for multiple testing ( $\mathrm{p}$-value $>0.05$ ).

To combine the SNPs located in the same gene, we performed a haplotype analysis for the IL10 [Supplementary data (Table II)] and TNF-BAT1-LTA [Supplementary data (Table III)] clusters. The results revealed no association between IL10 (rs1800890/rs1800896/ rs1800871) and TNF-BAT1-LTA (rs1800629/rs3853601/ rs909253/rs2239704) clusters with CCC outcome, as previously indicated for individual SNPs comparisons.

A linkage disequilibrium analysis for each of the genetic clusters (TGFB, IL10, and TNF-BAT-LTA) and evaluating the $C D$ patients recruited for the present study showed that, for the overall population of CD patients, $\mathrm{r}^{2}$ values were low $(<0.40)$, indicating no linkage disequilibrium among the markers in each of the clusters [Supplementary data (Fig. 2)].

TNF levels in the serum and TNF -308 genotype association - As expected, TNF levels in the serum were increased in $T$. cruzi-infected patients compared with those in non-infected controls putatively exposed to infection and born and residing in the same endemic areas (Fig. 1B). Although there was no correlation between TNF levels and reduction of LVEF (Fig. 1C), a significant increase in TNF levels in serum was detected in T. cruzi-infected patients of groups $\mathrm{B} 1$ and $\mathrm{C}$, compared with asymptomatic patients in group A (Fig. 1D). Nevertheless, our data showed no association between the $T N F$ rs1800629 -308 G>A genotype and TNF levels in serum of CD patients (Fig. 1E).

Polymorphisms at TNFR1 and TNFR2 genes - Considering that TNF signals via TNFR1 and TNFR2, we also analysed the possible influence of TNF receptors on CCC outcome. Gene variants of TNFR1 (rs767455, $+36 \mathrm{~A}>\mathrm{G}$ ) and TNFR2 (rs1061624, +1466 A $>\mathrm{G}$ ) showed no association with either development or progression of Chagas' heart disease in the studied groups (Table V).

Meta-analysis on the -308G $>A$ TNF gene and association with Chagas' heart disease - After a literature search, we observed that studies were not converging and that results for the association of $\mathrm{CD}$ and cytokine polymorphisms were controversial. We attempted to decrease ambiguity and searched for studies to conduct a meta-analysis. Selection of published articles for the meta-analysis resulted in 13 references that evaluated the same markers tested in the present study [Supplementary data (Fig. 2)]. Unfortunately, the reduced quantity of studies only allowed a meta-analysis of $T N F$ rs1800629 $-308 \mathrm{G}>\mathrm{A}(\mathrm{N}=5$ studies), comparing asymptomatic (A) vs. severe CCC (C) patients in Brazilian, Colombian, Mexican, and Peruvian populations (Beraún et al. 1998, Rodríguez-Pérez et al. 2005, Drigo et al. 2007, Pissetti et al. 2011, Criado et al. 2012), as summarised in Supplementary data (Table IV). After testing the inclusion/ exclusion criteria, it was verified that the asymptomatic group in the study by Rodríguez-Pérez et al. (2005) did not follow HWE, and therefore was excluded from further analysis. The forest plots for allele, carrier, and genotype comparisons (Fig. 2) showed that the OR values and confidence intervals were not statistically significant. Consensus values for the published studies for the random model effects analysis are detailed in Table $\mathrm{VI}$ and depict a risk association for $\mathrm{A}$ allele $(\mathrm{OR}=1.7$, $\mathrm{p}$-value $=0.02)$ and carriers of the minor allele $\mathrm{A}(\mathrm{OR}=$ 2.1, p-value $=0.02$ ). However, when the current study was included in the meta-analysis (group A, asymptomatic, 110 patients; group $\mathrm{C}$, severe CCC, 133 patients), reaching a total of $534 \mathrm{CCC}$ and 472 asymptomatic patients, the association described above did not retain significance for A allele or A carriers ( $\mathrm{p}$-values $=0.14$ and 0.15, respectively), as shown in Table VI and Fig. 2.

\section{DISCUSSION}

Out of the 13 cytokine-related polymorphisms that were studied for susceptibility to or progression of CCC, only BAT1 $-22 \mathrm{C}>\mathrm{G}$ (rs3853601) and IFNG +874 T>A (rs2430561) remained borderline significant for protection in the logistic regression analysis with adjustment for nongenetic covariates. Neither TGFB rs8179181, rs8105161, rs1800469), IL10 (rs1800890/rs1800896/rs1800871), nor TNF-BAT1-LTA (rs1800629/rs3853601/rs909253/rs2239704) analysed haplotypes displayed significant associations with Chagas' heart disease outcome.

The genetic variants in TGFB1 $-509 \mathrm{C}>\mathrm{T}$ (rs1800469) and $+10 \mathrm{~T}>\mathrm{C}$ (rs1800470) have been associated with susceptibility to T. cruzi infection, but not to CCC, in Colombians and Peruvians (Calzada et al. 2009). However, we were not able to corroborate these results in the $T G F B$ cluster $(+20743 \mathrm{C}>\mathrm{T},+19318 \mathrm{~A}>\mathrm{G},-509 \mathrm{C}>\mathrm{T})$, when searching for an association with risk of or progression to severe CCC. Regarding the regulatory cytokine IL-10, a study in Brazilians showed that $I L-10-1082$ A allele was correlated with low IL-10 expression and CCC susceptibility (Costa et al. 2009). Conversely, as previously shown in a Colombian population (Flórez et al. 2011), the present study on CD patients born and resident at the northeast of Brazil showed no association of alleles and genotypes in the $I L-10$ cluster with clinical outcome of CD.

In Colombians, the IFNG $+874 \mathrm{~T}$ allele has been associated with risk of developing $\mathrm{CD}$ per se but not CCC 


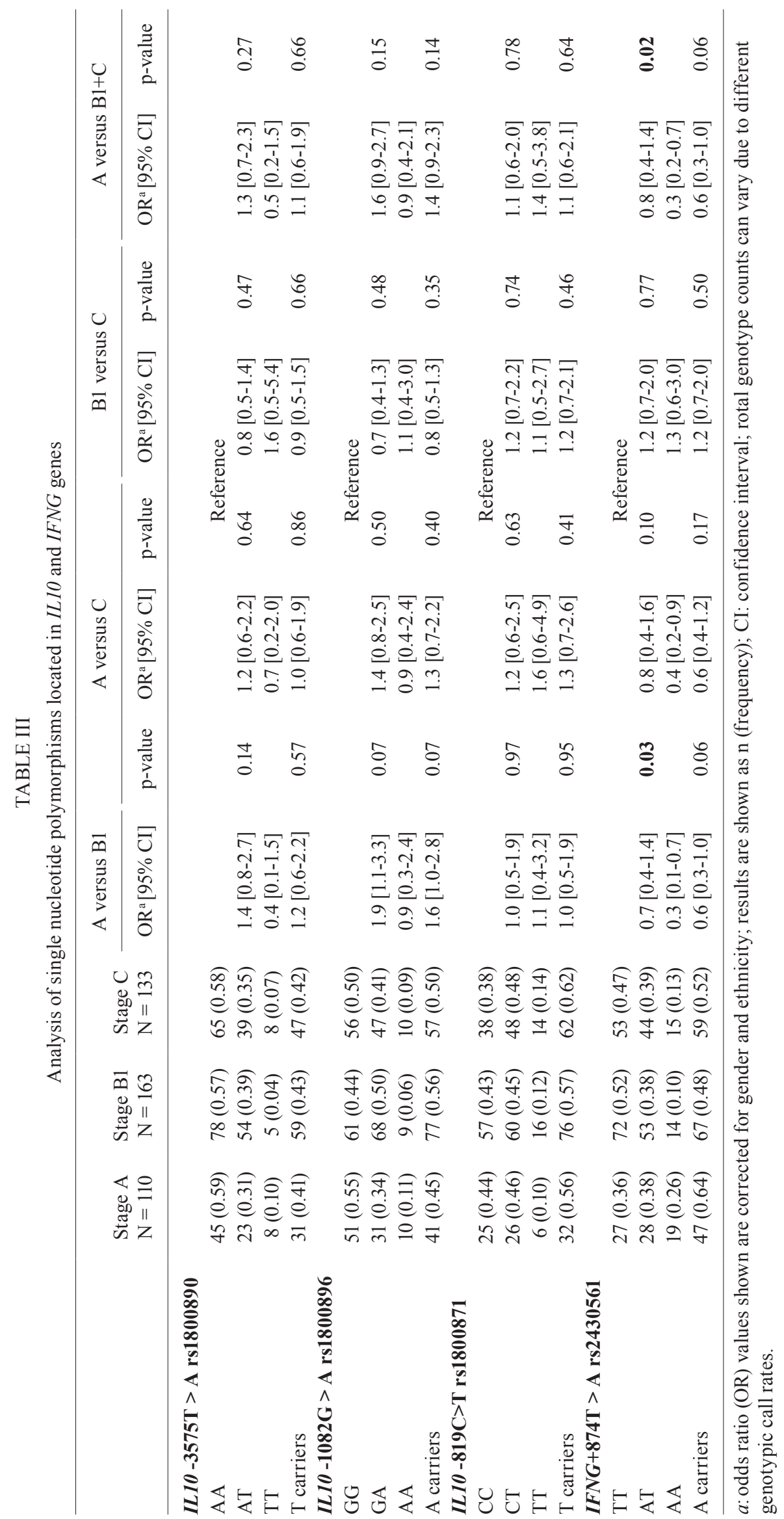




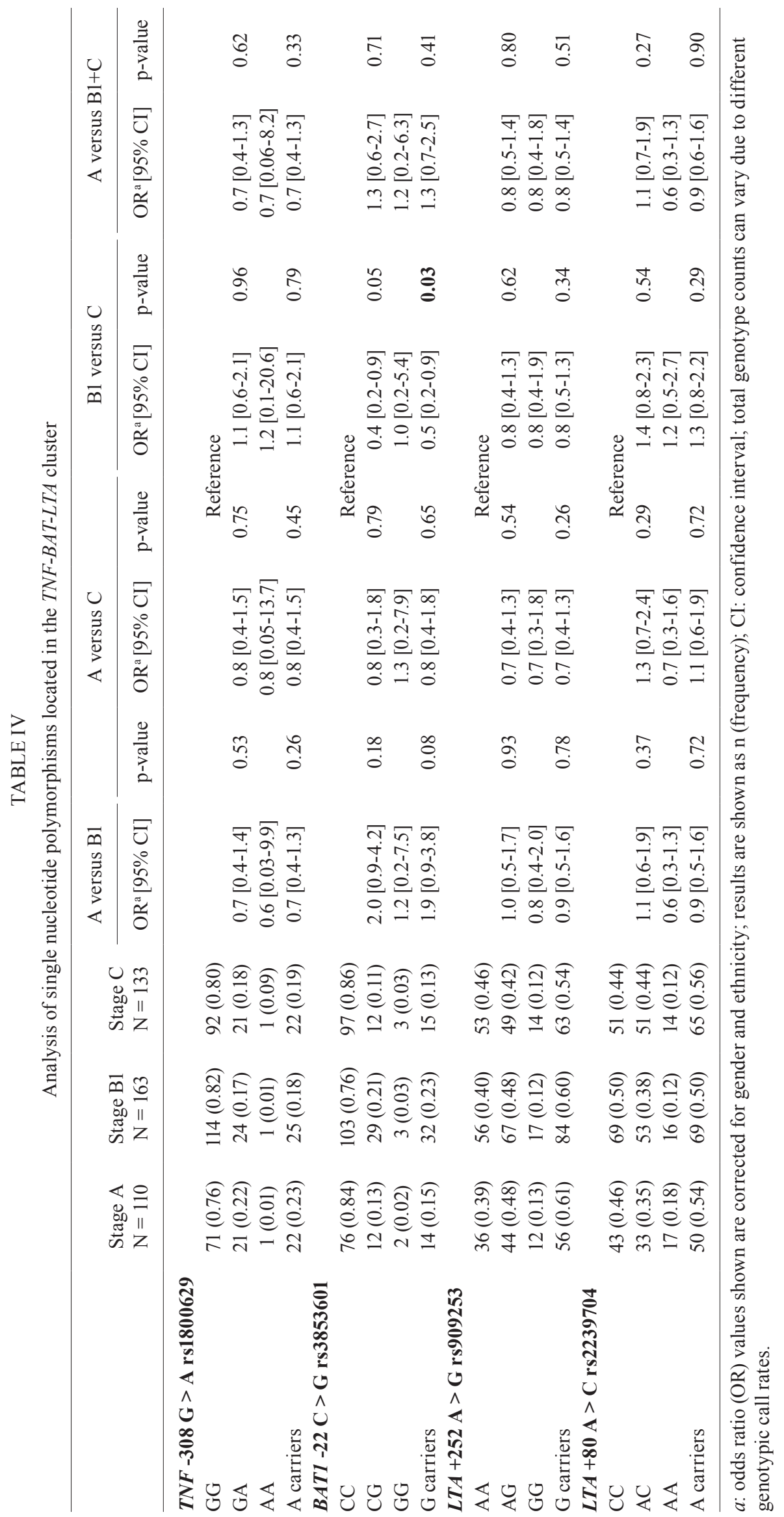




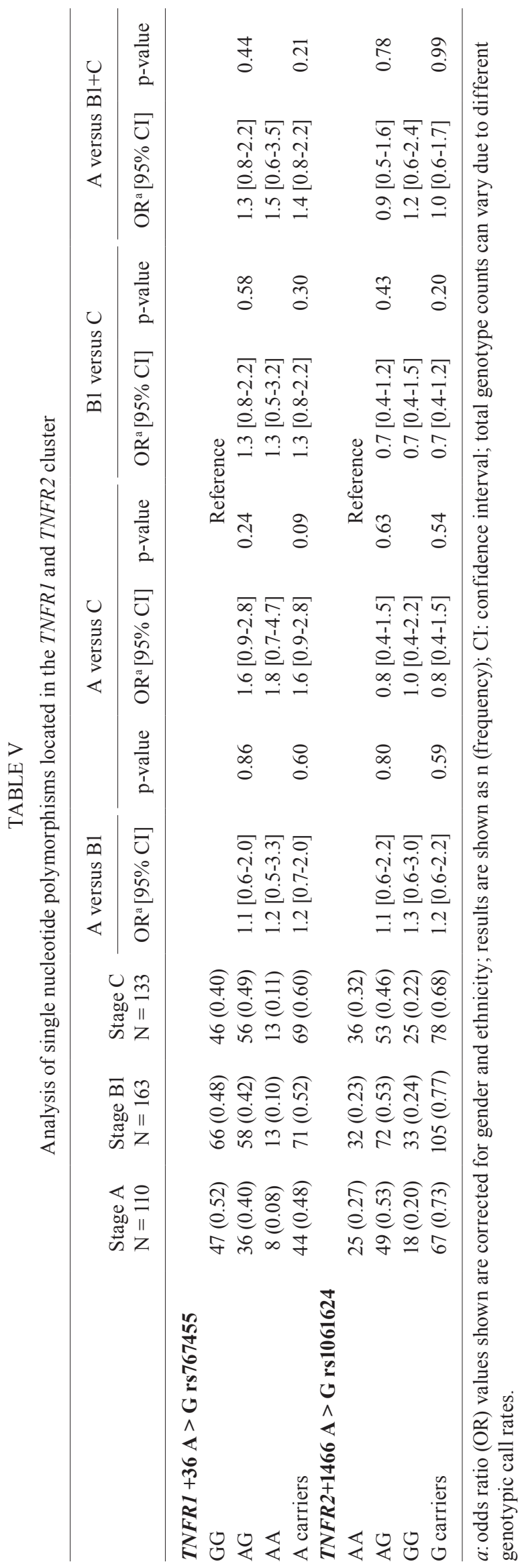

(Torres et al. 2010). Interestingly, in the present study, we observed an association with protection of developing the cardiac form of disease in the genotype comparison (A vs. B1, $\mathrm{p}=0.03$; $\mathrm{A} v s . \mathrm{B} 1+\mathrm{C}, \mathrm{p}=0.02$ ), and a borderline result for this protective effect for carriers of the $\mathrm{A}$ allele $(\mathrm{A} v s . \mathrm{B} 1$ and $\mathrm{A} v s . \mathrm{B} 1+\mathrm{C}, \mathrm{p}=0.06)$. These discrepant findings may be the result of a difference in genotypic distributions between Brazilian and Colombian populations. In our setting, the Brazilian CD patients present $\mathrm{AA}$ as the minor genotype $($ Freq $=0.16)$, whereas, in patients from Colombia, this is reversed, with TT as the minor genotype $($ Freq $=0.11)$. Thus, these data indicate that if we maintained the same baseline for comparison (e.g., TT) then both studies would point to an association with protection, therefore, suggesting that the A allele associated with lower IFN $\gamma$ production could be acting as a beneficial factor in infection, and, in our case, be associated with progression to milder forms of Chagas' heart disease. Interestingly, low IFN $\gamma$ serum levels were detected in $\mathrm{CD}$ patients with the indeterminate form and high IFN $\gamma$ concentrations associated with Chagas' heart disease (Sousa et al. 2014). Further, IFN $\gamma$-inducible genes were upregulated in cardiac tissue from CCC patients (Cunha-Neto and Chevillard 2014); however, murine model studies suggest a higher level of complexity for the role of cytokines in chagasic cardiomyopathy. In acute experimental T. cruzi-infection, IFN $\gamma$ is essential for parasite control (Aliberti et al. 2001). Nevertheless, in chronic infection, IFN $\gamma$ may fuel $T$. cruzi invasion of host cells and parasite persistence in the central nervous tissue (Silva et al. 2015). Most intriguing, T. cru$z i$-specific $\mathrm{CD}^{+} \mathrm{IFN} \gamma^{+} \mathrm{T}$ cells, mostly accumulated in peripheral tissues (blood and spleen), play a protective role against tissue injury when able to migrate towards lesioned myocardium (Silverio et al. 2012).

In the present study, the TNF -308 (rs1800629) polymorphism showed no significant association with risk of or progression to severe Chagas' heart disease. Polymorphisms in the TNF gene were previously evaluated in populations from Peru, Mexico, Brazil, and Colombia, and results showed no consensus. In a study in Peruvian CD patients, markers at -308 (rs1800629), -244, and -238 (rs361525) showed no associations, when comparing asymptomatic vs. the CCC group. On the other hand, the TNF -308A allele showed a risk association, considering both CD disease per se and CCC susceptibility in Mexicans (Rodríguez-Pérez et al. 2005). In Brazilians, 42 CCC patients that were A allele carriers had a reduced mean survival time compared with non-carriers (Drigo et al. 2005). Unfortunately, the same group was not able to replicate this association in a later study (Drigo et al. 2007). Another study suggested a susceptibility association between CCC and TNF -238 SNP, but not TNF -308 (Pissetti et al. 2011). A more recent study in Colombians also included polymorphism TNF $-1031 \mathrm{C}>\mathrm{T}$ (rs1799964) in the equation, showing that $\mathrm{C}$ allele carriers as well as $-308 \mathrm{~A}$ carriers displayed a significant association with CCC (Criado et al. 2012). TNF signals via TNF receptor 1 (TNFR1/p55) and TNFR2 (p75). TNFR1, but not TNFR2, has been shown to control acute $T$. cruzi infection (Aliberti et al. 2001). Re- 
10|12 Cytokine-related genes in Chagas disease - Lucia Elena Alvarado-Arnez et al.

TABLE VI

Summary of meta-analysis of published studies for TNF -308 G > A (rs1800629) and chagasic cardiomyopathy ${ }^{a}$

\begin{tabular}{|c|c|c|c|c|c|c|c|c|}
\hline \multirow[b]{2}{*}{$T N F-308 \mathrm{G}>$ A rs1800629 } & \multicolumn{4}{|c|}{ Meta-analysis of published studies } & \multicolumn{4}{|c|}{ Meta-analysis of published studies and present data } \\
\hline & $\begin{array}{c}\mathrm{CCC} \\
\mathrm{N}=424\end{array}$ & $\begin{array}{c}\text { ASY } \\
N=339\end{array}$ & $\mathrm{OR}^{b}[95 \% \mathrm{CI}]$ & p-value & $\begin{array}{c}\mathrm{CCC} \\
\mathrm{N}=534\end{array}$ & $\begin{array}{c}\text { ASY } \\
\mathrm{N}=472\end{array}$ & $\mathrm{OR}^{b}[95 \% \mathrm{CI}]$ & p-value \\
\hline Allele A & 124 & 60 & $1.7[1.1-2.6]$ & 0.02 & 147 & 83 & $1.4[0.9-2.3]$ & 0.14 \\
\hline A carriers & 117 & 56 & $2.1[1.1-3.1]$ & 0.02 & 139 & 78 & $1.5[0.9-2.6]$ & 0.15 \\
\hline AA Genotype & 7 & 4 & $1.3[0.4-4.5]$ & 0.70 & 8 & 5 & $1.2[0.4-3.7]$ & 0.77 \\
\hline
\end{tabular}

$a$ : results are shown for random-effects model; $b$ : odds ratio (OR); CCC: chronic chagasic cardiomyopathy; ASY: asymptomatic group; CI: confidence interval; the study by Rodríguez-Pérez et al. (2005) was excluded due to deviations from HWE in the control group.
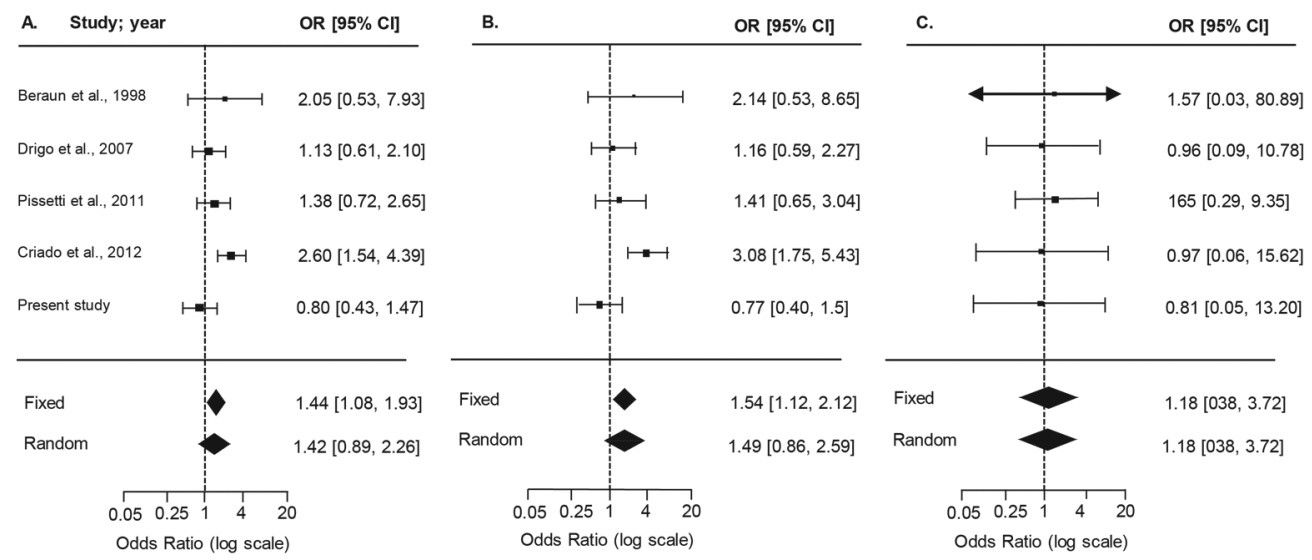

Fig. 2: forest plots summarising the association between the $T N F-308 \mathrm{G}>\mathrm{A}$ SNP and susceptibility to Chagas' heart disease, based on allele, carrier, and genotype comparisons. Results from the random-effects model are shown. Bars represent $95 \%$ confidence intervals (CIs) and boxes represent the odds ratio (OR) values. The size of each box indicates the weight of the study in the pooled results.

cently, TNF/TNFR1 signalling has been associated with increased TNF production and cardiac tissue damage (Vilar-Pereira et al. 2016). A previous report indicated that the TNFR2 $+676 \mathrm{G}>\mathrm{T}$ SNP, which was associated with lower TNFR2 expression, was monomorphic in the Colombian population (Criado et al. 2012). In our study neither TNFR1 +36 A $>\mathrm{G}$ (rs767455) nor TNFR2 +1466 $A>G$ (rs1061624) showed a significant association with Chagas' heart disease outcome.

In addition to $T N F$, the chromosome 6p21 region encompasses other genes such as LTA and BAT1 that have been evaluated in CD. LTA has a pro-inflammatory role and shares the same receptors as TNF (TNFR1 and TNFR2). BAT1 -22 C>G (rs3853601) and $-348 \mathrm{C}>\mathrm{T}$ variants, which alter the transcriptional activity of the gene itself, have been linked to CCC development (Ramasawmy et al. 2006). In CD, the LTA $+252 \mathrm{G}$ (rs909253) and $+80 \mathrm{C}$ (rs2239704) alleles independently or as a haplotype combination were associated with CCC susceptibility in a population composed of individuals from different geographical regions in Brazil (Ramasawmy et al. 2007). Moreover, in another Brazilian study, patients from the state of Minas Gerais showed a 2.8 times higher risk of developing CCC in LTA $+252 \mathrm{GG}$ genotype carriers, and the $\mathrm{G}$ allele was associated with higher LTA production (Pissetti et al. 2013). However, in the present study, focused on a population born and residing in the state of Pernambuco in north-eastern Brazil, we were not able to corroborate these findings.

The correlation between higher TNF levels and worsening of CCC, stablished by a reduction in LVEF, has been described (Sousa et al. 2014). In our study, although TNF serum levels were increased in cardiopathic B1 and C patients compared with asymptomatic A patients, there was no correlation between TNF levels and decrease of LVEF. Functional characterisation of cytokine production and its association with disease phenotypes or with the polymorphism genotypes represents a challenge in $\mathrm{CD}$. As previously stated, the complex structure of 6 p21 could suggest that polymorphic sites other than -308 (rs1800629) affect TNF levels (Ramasawmy et al. 2007). A meta-analysis of functional studies focusing on the influence of -308 on TNF levels showed no effect at the mRNA level (Mekinian et al. 2011). Indeed, our study reveals no association between TNF expression and the TNF -308 (rs1800629) genotype. Altogether, these findings suggest that TNF may be merely a component of a complex inflammatory profile associated with cardiomyopathy in $\mathrm{CD}$, rather than a single determinant factor.

Although it is tempting to suggest the possible influence of BAT1 -22 (rs3853601) and IFNG +874 (rs2430561) polymorphisms in Chagas' heart disease outcomes in the present work, the interactions with other cytokines and their genetic variants could also participate in deter- 
mining these outcomes. In fact, $M I F, I L 1 B, I L 1 R N, I L 4$, and $I L 12$ SNPs also showed an association with disease susceptibility and CCC progression in Latin American CD populations (Ayo et al. 2013). Further, the apparent association with high/low producing alleles is not straightforward when translated to functional cytokine levels, and this should be studied further. Additionally, one should keep in mind that in $\mathrm{CD}$, cytokine production may be a consequence of more complex parasite/ host interplay. In $\mathrm{CD}$, the fine balance that controls the pathogen and sustains an efficient immunologic response may rely on host genetic and non-genetic factors. The complex interaction with the parasite has proven to be relevant, as different $T$. cruzi strains have been associated with differential chronic outcomes (Cura et al. 2012, Rassi Jr et al. 2017).

Because of such results, we performed a literature search of the SNPs evaluated in our study. The number of studies on CD was low; therefore, we could only carry out a meta-analysis of TNF studies with the objective of attaining a consensus in OR estimates. In the Mexican study by Rodríguez-Pérez et al. (2005), the control group (asymptomatic) did not follow HWE assumptions and therefore was excluded from analysis. Our summary results initially showed a significant risk association for -308 (rs1800629) A allele and A carriers. After inclusion of our study, although the number of individuals was increased to 534 for CCC and 472 for asymptomatic patients, there were no consensus in the pooled OR estimates, and the significance from previous comparisons was lost. This could be because of the different clinical classifications that each study considered in determining patients asymptomatic and having CCC. In addition, the number of published articles on TNF -308 (rs1800629) gene polymorphisms in CD is low compared to studies on other chronic infectious diseases such as tuberculosis, for which more than 15 studies were available (Wang et al. 2012). Therefore, these data reinforce the need of more independent association studies in CD that evaluate the clinical associations with this and other polymorphisms, as well as the importance of standardising clinical outcomes for $\mathrm{CD}$ patients.

One limitation of most of the studies on gene polymorphisms in CD is sample size, and we also consider this feature a limitation in our study, with borderline association results impacted by adjustment for multiple testing. However, we show that the sample evaluated in the present work represents a population that is geographically distinct from those in previously published literature on $\mathrm{CD}$ and genetic association studies. In addition, a relevant point in our study is the strict clinical classification of CD patients. For decades, the criteria defining the different stratification and prognostic tests for the chronic phase remained highly debatable. In 2009, a systematic review highlighted three principal prognostic estimators: functional group according to the New York Heart Association, cardiac enlargement (cardiomegaly), and a reduction in the left ventricle ejection fraction (\% LVEF) (Rassi Jr and Rassi 2010). To normalise the stratification in clinical groups of CCC patients, after consensus, the I Latin American Guideline for the Diagnosis and Treatment of Chagas' Heart Disease was published (Andrade et al. 2011). These standardised parameters and functional groups were adopted in the present study. Therefore, differences in clinical characterisations could have reduced the homogeneity of the groups under comparison in the different studies included in the meta-analysis.

Regarding the $T N F-308$ (rs1800629) polymorphism, and considering the described results, it seems that the region represents complexity that it is not well resolved in recent studies. The $T N F$ gene is contained in the telomeric portion adjacent to HLA III. Many SNPs in this region have been shown to be in linkage disequilibrium with HLA II. In fact, HLA-DRB1 alleles were associated with CCC susceptibility in several populations (WilliamsBlangero et al. 2011, Ayo et al. 2013). Overall, it seems that this chromosomic region requires a higher density of markers and testing with larger study populations.

Potential polymorphisms that could serve as prognostic markers to indicate groups prone to developing severe cardiac manifestations of CD would be of interest. This is especially true for this disease, considering the long period of time for presentation of clinical symptoms of progression. The genetic, functional, and systematic review strategies explored in our study pointed to some borderline genetic associations. Overall, our study indicates the difficulty of characterising the influence of genetic variants localised in immune response genes relevant to $\mathrm{CD}$ outcome. The absence of a strong genetic biomarker may indicate that the consequences of an immune response, including parasite control and the inflammatory profile, for $\mathrm{CD}$ patients are not genetically predetermined. Therefore, this lack of evidence may stimulate further studies and support the search for physiopathological factors resulting from parasite/host interactions that contribute to Chagas' heart disease outcome and severity. This approach may lead to therapeutic strategies to modify this interplay, ameliorating the prognosis and improving the quality of life for $\mathrm{CD}$ patients.

\section{ACKNOWLEDGEMENTS}

To all of the patients that voluntarily participated in the study, all the staff of the House of Chagas (Casa de Chagas), and the Association of Chagas disease patients of Pernambuco for their collaboration during patient recruitment and clinical evaluation. We also acknowledge the invaluable contribution of nurse Valdinete Paiva (in memoriam) during recruitment, clinical evaluation, and patient care. The authors thank Ms Thayse do Espírito Santo Protásio da Silva and Rafael Rodrigues Silva for helping with DNA preparation and ELISA procedures, respectively. The authors also thank to the Program for Technological Development in Tools for Health (PDTIS-Fiocruz) for use of a real-time PCR platform (RPT09B).

\section{AUTHORS' CONTRIBUTION}

JLV, MOM and WOJr - Conception of the idea; JLV, MOM, WOJr, CC, LEAA and AMB - experimental design; LEAA, AMB, SMA, GM, VMBL and IRP - performing experiments; LEAA, AMB, SMA, GM, CCC, IRP, WOJr, MOM, AGP and JLV - data analysis; LEAA, AMB, MOM and JLV - writing. 


\section{REFERENCES}

Aliberti JC, Souto JT, Marino AP, Lannes-Vieira J, Teixeira MM, Farber J, et al. Modulation of chemokine production and inflammatory responses in interferon-gamma and tumor necrosis factor-R1-deficient mice during Trypanosoma cruzi infection. Am J Pathol. 2001; 158(4): 1433-40.

Andrade JP, Marin Neto JA, Paola AA, Vilas-Boas F, Oliveira GM, Bacal F, et al. I Latin American Guidelines for the diagnosis and treatment of Chagas' heart disease: executive summary. Arq Bras Cardiol. 2011; 96(6): 434-42.

Ayo CM, Dalalio MM, Visentainer JE, Reis PG, Sippert EÂ, Jarduli LR, et al. Genetic susceptibility to Chagas disease: an overview about the infection and about the association between disease and the immune response genes. Biomed Res Int. 2013; 2013: 284729.

Beraún Y, Nieto A, Collado MD, González A, Martín J. Polymorphisms at tumor necrosis factor (TNF) loci are not associated with Chagas' disease. Tissue Antigens. 1998; 52(1): 81-3.

Calzada JE, Beraún Y, González CI, Martín J. Transforming growth factor beta 1 (TGFbetal) gene polymorphisms and Chagas disease susceptibility in Peruvian and Colombian patients. Cytokine. 2009; 45(3): 149-53.

Costa GC, Rocha MOC, Moreira PR, Menezes CA, Silva MR, Gollob $\mathrm{KJ}$, et al. Functional IL-10 gene polymorphism is associated with Chagas disease cardiomyopathy. J Infect Dis. 2009; 199(3): 451-4.

Criado L, Flórez O, Martín J, González CI. Genetic polymorphisms in TNFA/TNFR2 genes and Chagas disease in a Colombian endemic population. Cytokine. 2012; 57(3): 398-401

Cunha-Neto E, Chevillard C. Chagas disease cardiomyopathy: immunopathology and genetics. Mediators Inflamm. 2014; 2014: 683230.

Cura CI, Lucero RH, Bisio M, Oshiro E, Formichelli LB, Burgos JM, et al. Trypanosoma cruzi discrete typing units in Chagas disease patients from endemic and non-endemic regions of Argentina. Parasitology. 2012; 139(4): 516-21.

Dias JC, Ramos Jr AN, Gontijo ED, Luquetti A, Shikanai-Yasuda MA, Coura JR, et al. 2nd Brazilian consensus on Chagas disease, 2015. Rev Soc Bras Med Trop. 2016; 49(Suppl. 1): 3-60.

Drigo SA, Cunha-Neto E, Ianni B, Cardoso MR, Braga PE, Faé KC, et al. TNF gene polymorphisms are associated with reduced survival in severe Chagas' disease cardiomyopathy patients. Microbes Infect. 2005; 8(3): 598-603.

Drigo SA, Cunha-Neto E, Ianni B, Mady C, Faé KC, Buck P, et al. Lack of association of tumor necrosis factor-alpha polymorphisms with Chagas disease in Brazilian patients. Immunol Lett. 2007; 108(1): 109-11.

Flórez O, Martín J, González CI. Interleukin 4, interleukin 4 receptor- $\alpha$ and interleukin 10 gene polymorphisms in Chagas disease. Parasite Immunol. 2011; 33(9): 506-11.

Herricks JR, Hotez PJ, Wanga V, Coffeng LE, Haagsma JA, Basáñez MG, et al. The global burden of disease study 2013: What does it mean for the NTDs? PLoS Negl Trop Dis. 2017; 11(8): e0005424.

Mekinian A, Tamouza R, Pavy S, Gestermann N, Ittah M, Mariette $\mathrm{X}$, et al. Functional study of TNF- $\alpha$ promoter polymorphisms: literature review and meta-analysis. Eur Cytokine Netw. 2011; 22(2): 88-102.

Moher D, Liberati A, Tetzlaff J, Altman DG, PRISMA Group. Preferred reporting items for systematic reviews and meta-analyses: the PRISMA statement. Ann Intern Med. 2009; 151(4): 264-9.
Pissetti CW, Correia D, de Oliveira RF, Llaguno MM, Balarin MA, Silva-Grecco RL, et al. Genetic and functional role of TNF-alpha in the development Trypanosoma cruzi infection. PLoS Negl Trop Dis. 2011; 5(3): e976.

Pissetti CW, de Oliveira RF, Correia D, Nascentes GA, Llaguno MM, Rodrigues Jr V. Association between the lymphotoxin-alpha gene polymorphism and chagasic cardiopathy. $\mathrm{J}$ Interferon Cytokine Res. 2013; 33(3): 130-5.

Ramasawmy R, Cunha-Neto E, Faé KC, Müller NG, Cavalcanti VL, Drigo SA, et al. BAT1, a putative anti-inflammatory gene, is associated with chronic Chagas cardiomyopathy. J Infect Dis. 2006; 193(10): 1394-9.

Ramasawmy R, Fae KC, Cunha-Neto E, Müller NG, Cavalcanti VL, Ferreira RC, et al. Polymorphisms in the gene for lymphotoxinalpha predispose to chronic Chagas cardiomyopathy. J Infect Dis. 2007; 196(12): 1836-43.

Rassi Jr A, Marin-Neto JA, Rassi A. Chronic Chagas cardiomyopathy: a review of the main pathogenic mechanisms and the efficacy of aetiological treatment following the BENznidazole Evaluation for Interrupting Trypanosomiasis (BENEFIT) trial. Mem Inst Oswaldo Cruz. 2017; 112(3): 224-35.

Rassi Jr A, Rassi A. Predicting prognosis in patients with Chagas disease: why are the results of various studies so different? Int $\mathrm{J}$ Cardiol. 2010; 145(1): 64-5.

Rodríguez-Pérez JM, Cruz-Robles D, Hernández-Pacheco G, PérezHernández N, Murguía LE, Granados J, et al. Tumor necrosis factor-alpha promoter polymorphism in Mexican patients with Chagas' disease. Immunol Lett. 2005; 98(1): 97-102.

Saraiva RM, Waghabi MC, Vilela MF, Madeira FS, da Silva GMS, Xavier SS, et al. Predictive value of transforming growth factor$\beta 1$ in Chagas disease: towards a biomarker surrogate of clinical outcome. Trans R Soc Trop Med Hyg. 2013; 107(8): 518-25.

Silva RR, Mariante RM, Silva AA, dos Santos AL, Roffê E, Santiago $\mathrm{H}$, et al. Interferon-gamma promotes infection of astrocytes by Trypanosoma cruzi. PLoS One. 2015; 10(2): e0118600.

Silverio JC, Pereira IR, Cipitelli MC, Vinagre NF, Rodrigues MM, Gazzinelli RT, et al. CD8+ T-cells expressing interferon gamma or perforin play antagonistic roles in heart injury in experimental Trypanosoma cruzi-elicited cardiomyopathy. PLoS Pathog. 2012; 8(4): e1002645.

Sousa GR, Gomes JA, Fares RC, Damásio MP, Chaves AT, Ferreira $\mathrm{KS}$, et al. Plasma cytokine expression is associated with cardiac morbidity in Chagas disease. PLoS One. 2014; 9(3): e87082.

Torres OA, Calzada JE, Beraún Y, Morillo CA, González A, González $\mathrm{CI}$, et al. Role of the IFNG $+874 \mathrm{~T} / \mathrm{A}$ polymorphism in Chagas disease in a Colombian population. Infect Genet Evol. 2010; 10(5): 682-5.

Vilar-Pereira G, Pereira IR, Ruivo LAS, Moreira OC, da Silva AA, Britto $\mathrm{C}$, et al. Combination chemotherapy with suboptimal doses of benznidazole and pentoxifylline sustains partial reversion of experimental Chagas' heart disease. Antimicrob Agents Chemother. 2016; 60(7): 4297-309.

Wang Q, Zhan P, Qiu LX, Qian Q, Yu LK. TNF-308 gene polymorphism and tuberculosis susceptibility: a meta-analysis involving 18 studies. Mol Biol Rep. 2012; 39(4): 3393-400.

Williams-Blangero S, VandeBerg JL, Blangero J, Corrêa-Oliveira R. Genetic epidemiology of Chagas disease. Adv Parasitol. 2011; 75: $147-67$. 